\title{
Incidence and predictive factors of lower respiratory tract infections among the very elderly in the general population. The Leiden 85-plus Study
}

\author{
A Sliedrecht, ${ }^{1,2}$ W P J den Elzen, ${ }^{2}$ T J M Verheij, ${ }^{1}$ R G J Westendorp, ${ }^{3}$ J Gussekloo ${ }^{2}$
}

1 Julius Centre for Health Sciences and Primary Care, University Medical Centre Utrecht, Utrecht, The Netherlands: ${ }^{2}$ Department of Public Health and Primary Care, Leiden University Medical

Centre, Leiden, The Netherlands;

${ }^{3}$ Department of Gerontology and Geriatrics, Leiden University Medical Centre, Leiden, The Netherlands

Correspondence to: Dr J Gussekloo, Department of Public Health and Primary Care, Leiden University Medical Center, Post zone V-O-P, PO Box 9600, 2300 RC Leiden, The Netherlands; jgussekloo@ lumc.nl

Received 3 November 2007 Accepted 13 February 2008 Published Online First 3 April 2008

\begin{abstract}
Objectives: To target preventive strategies in old age, which of the very elderly are predisposed to developing lower respiratory tract infections was investigated.

Design: Prospective observational follow-up study.

Setting: General population.

Participants: Unselected cohort of 587 participants aged 85 years in Leiden, The Netherlands.

Measurements: As reported in the literature, predictive factors were selected and assessed at baseline. During a 5 year follow-up period, information on the development of lower respiratory tract infections was obtained from general practitioners or nursing home physicians. Associations between predictive factors were analysed with Cox regression, and population attributable risks were calculated.
\end{abstract}

Results: The incidence of lower respiratory tract infections among persons aged 85-90 years was 94 (95\% Cl 80-108) per 1000 person years. After multivariate analysis, history of chronic obstructive pulmonary disease (COPD), smoking, oral glucocorticosteroid use, severe cognitive impairment, history of stroke and declined functional status remained independently associated with the occurrence of lower respiratory tract infections. Smoking was the greatest contributor with a population attributable risk of $32 \%$.

Conclusion: In the very old, smoking, COPD, stroke and declined functional status were associated with the occurrence of lower respiratory tract infections and provide a means of targeting patients at risk of severe health complications.

In the very elderly, the impact of lower respiratory tract infections is high, not only because of a high incidence, but also because elderly patients are likely to develop medical complications, be admitted to hospital or die from lower respiratory tract infections. ${ }^{1-5}$ As the incidence of lower respiratory tract infections increases with age, ${ }^{12} 6$ timely diagnosis and treatment of lower respiratory tract infections and preventive strategies against its occurrence, for instance pneumococcal vaccination, ${ }^{7-10}$ should be emphasised.

For introducing these preventive strategies, it is important to know which subgroups of the very elderly population are predisposed to developing lower respiratory tract infections. Earlier studies have shown that increasing age, ${ }^{34}$ institutionalisation, ${ }^{45}$ smoking, ${ }^{3}{ }^{511}$ alcoholism, ${ }^{4}$ pulmonary disease, ${ }^{4511}$ heart disease, ${ }^{45}$ neurological disease ${ }^{5}$ and immunosuppressive therapy ${ }^{4}$ may be contributing factors in the susceptibility of individuals to lower respiratory tract infections. However, until now, no studies have been performed among the very elderly in the general population among whom the incidence is highest. Most previous studies that have investigated factors associated with lower respiratory tract infections included hospitalised patients and concerned the prognosis of patients with community acquired pneumonia only. ${ }^{12-20}$

The purpose of this study was to determine the incidence and predictive factors of lower respiratory tract infections among persons aged 85 years and over in the general population in order to provide more insight into which subgroups of the very elderly are at the highest risk of developing lower respiratory tract infections.

\section{METHODS}

\section{Setting and study population}

The Leiden 85-plus Study is an observational population based prospective study of 85 -year-old inhabitants of Leiden, The Netherlands. The overall aim was to investigate determinants and preventable causes of unsuccessful aging and to explore the possibilities for investing in successful aging.

Between September 1997 and September 1999, all inhabitants of Leiden who reached the age of 85 years were invited to participate in the study. There were no selection criteria concerning health or demographic characteristics. Participants were followed for 5 years until the age of 90 years or until death. At baseline, all participants were visited at their place of residence where face to face interviews were conducted, cognitive testing was performed, information on income, education and disabilities in daily activities was obtained, and a venous blood sample was drawn.

All participants gave informed consent; for people who were severely cognitively impaired, a guardian gave informed consent. The Medical Ethics Committee of Leiden University Medical Centre approved the study.

Dates of death were obtained from the municipality. Specific data on causes of death were obtained from Statistics Netherlands, according to the International Classification of Diseases and Related Disorders, 10th revision (ICD-10). The assignment of causes of death was done independently of the risk factors that were determined at age 85 years.

\section{Lower respiratory tract infection}

The endpoint of this study was the development of the first lower respiratory tract infection during the follow-up period. This endpoint was considered 
present when a treating general practitioner or nursing home physician diagnosed a lower respiratory tract infection based on history taking, physical examination and clinical judgement. These data were gathered during annual interviews with participating physicians. The endpoint was also reached when a participant without a clinical diagnosis of lower respiratory tract infection during follow-up died from pneumonia.

\section{Selection of potentially predictive factors}

Based on a search of the medical literature, ${ }^{124-611-13}$ factors potentially associated with lower respiratory tract infections in the elderly were identified and selected for study. They were categorised as sociodemographic factors, functioning, comorbidities and medication use. All potentially predictive factors were assessed when participants enrolled in the study at the age of 85 years.

\section{Sociodemographic factors}

During baseline interviews, a research nurse collected information on participants' residency, income, level of education and smoking habits.

\section{Functioning}

To assess cognitive function, the Mini-Mental State Examination (MMSE) was used. Severe cognitive impairment was defined as a MMSE score below 19 points. ${ }^{21}$ In order to determine the presence of depressive symptoms, the Geriatric Depression Scale-15 (GDS-15) was performed; depressive symptoms were considered present in cases where a GDS score was above 4 points. The GDS-15 was only administered to participants with sufficient cognitive function (that is, to those with an MMSE score of 19 points or higher). ${ }^{21}$ Disability in

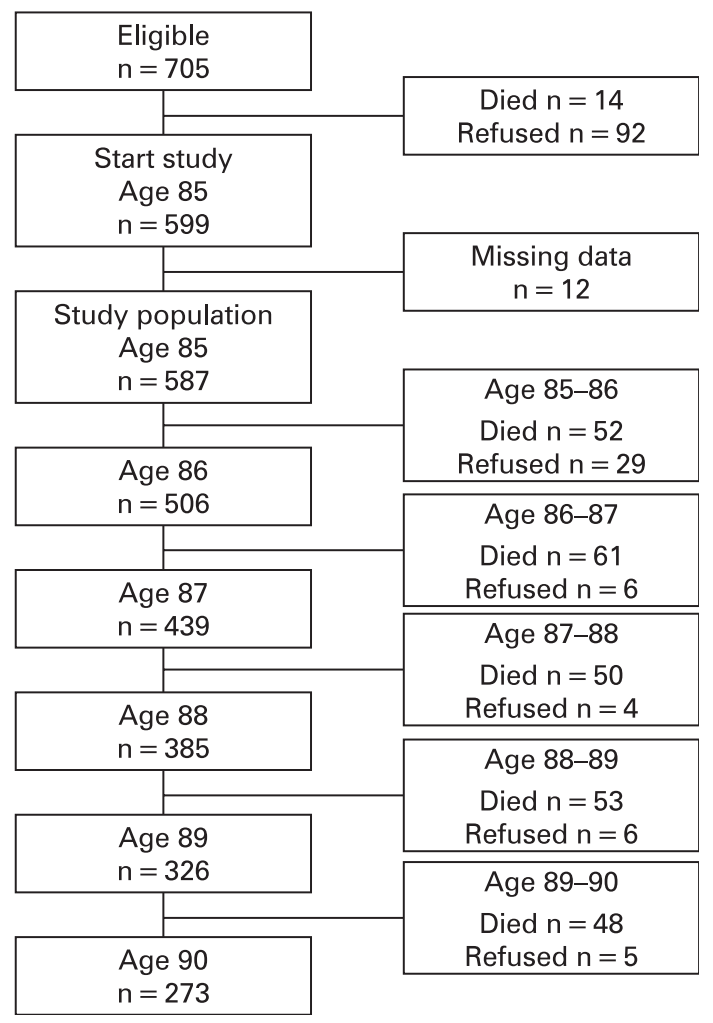

Figure 1 Number of participants aged 85-90 years in the Leiden 85-plus Study. basic activities of daily life was determined using the Groningen Activity Restriction Scale. We defined disability as being unable to do any one of the following nine basic activities of daily life independently: walk inside, get out of bed, get into and out of a chair, use the toilet, wash hands and face, wash body, dress and undress, eat and drink, and make breakfast. ${ }^{21}$

\section{Comorbidities and medication use}

Information on participants' medical history was obtained by standardised interviews with their treating general practitioner or nursing home physician. Information on participants' use of medication was obtained by interviewing their pharmacists. We obtained clinical information on the presence of diabetes mellitus, hypertension, myocardial infarction, stroke, heart failure and cancer. The presence of chronic obstructive pulmonary disease (COPD) was considered present when the diagnosis of COPD was reported by the treating physician or when lung medication (Anatomical Therapeutic Chemical (ATC) code R03) was used at age 85 years. Information on the use of glucocorticosteroids was obtained from the participants' pharmacists and was also coded according to the ATC classification. ${ }^{22}$ The presence of anaemia was based on baseline haemoglobin level, which was determined by an automated system (Coulter Counter, Coulter Electronics, Hialeah, Florida, USA). Anaemia was defined according to WHO criteria: haemoglobin $(\mathrm{Hb})<7.5 \mathrm{mmol} / \mathrm{l}$ for women and $\mathrm{Hb}$ $<8.1 \mathrm{mmol} / 1$ for men. ${ }^{23}$ Creatinine clearance and urea were determined as measures of renal function. Serum creatinine concentration was measured automatically according to the Jaffe method (Hitachi 747; Hitachi, Tokyo, Japan). Creatinine

Table 1 Baseline characteristics of the study population at age 85 years $(n=587)$

\begin{tabular}{|c|c|}
\hline & n (\%) \\
\hline \multicolumn{2}{|l|}{ Sociodemographic factors } \\
\hline Male & $199(34)$ \\
\hline Institutionalised* & $104(18)$ \\
\hline Minimum income & $93(16)$ \\
\hline Primary school only & $380(65)$ \\
\hline Smoking (current or previous) & $278(48)$ \\
\hline No yearly influenza vaccine before 85 years old & $133(24)$ \\
\hline \multicolumn{2}{|l|}{ Functioning } \\
\hline Severe cognitive impairment (MMSE <19) & $96(16)$ \\
\hline Depressive symptoms (GDS-15>4) & $75(15)$ \\
\hline Disability in daily living $\dagger$ & $201(34)$ \\
\hline \multicolumn{2}{|l|}{ Comorbidities and medication use } \\
\hline Diabetes mellitus & $85(15)$ \\
\hline Hypertension & 226 (39) \\
\hline Myocardial infarction & $62(11)$ \\
\hline Stroke & $59(10)$ \\
\hline Heart failure & $74(13)$ \\
\hline Chronic obstructive pulmonary disease & $69(12)$ \\
\hline Cancer & $104(18)$ \\
\hline Anaemiat: & $149(27)$ \\
\hline Oral glucocorticosteroid use & $11(2)$ \\
\hline Creatinine clearance $<30 \mathrm{ml} / \mathrm{min}$ & $41(8)$ \\
\hline Urea $>7.5 \mathrm{mmol} / \mathrm{l}$ & $233(42)$ \\
\hline $\mathrm{CRP}>5 \mathrm{mg} / \mathrm{l}$ & $188(34)$ \\
\hline
\end{tabular}

*Institutionalised refers to nursing or retirement home resident.

†Disability in daily living = unable to do any one of nine basic activities of daily living independently, according to the Groningen Activity Restriction Scale $\$ \mathrm{Hb}_{\text {female }}<7.5 \mathrm{mmol} / \mathrm{l}, \mathrm{Hb}_{\text {male }}<8.1 \mathrm{mmol} / \mathrm{l}$.

CRP, C reactive protein; GDS-15, Geriatric depression scale with 15 items (only administered to participants with MMSE score $\geqslant 19(n=491)) ;$ MMSE, Mini-Mental State Examination. 
clearance was calculated from serum creatinine concentration and body weight, using the Cockcroft-Gault formula. ${ }^{24}$ Low creatinine clearance was defined as creatinine clearance $<30 \mathrm{ml} / \mathrm{min}$. Urea was measured in serum by means of a colorimetric method. Urea levels $>7.5 \mathrm{mmol} / 1$ were considered elevated. To further assess general health status, we determined $\mathrm{C}$ reactive protein (CRP) levels with a fully automated Hitachi 911 analysis system. CRP levels $>5 \mathrm{mg} / \mathrm{l}$ were considered elevated.

\section{Data analyses}

The incidence of lower respiratory tract infections from age 85 years onwards was calculated over 5 years of follow-up using the life table method. The number of first time lower respiratory tract infections was assigned to the nominator and the observed person years at risk was assigned to the denominator.

Factors which predicted the occurrence of lower respiratory tract infections in participants after the age of 85 years were investigated with Cox regression proportional hazard models. Those factors associated with lower respiratory tract infections in the univariate Cox regression analysis with a $p$ value below 0.10 were included in a multivariate Cox regression model. Since the GDS-15 was only administered to participants without cognitive impairment (an MMSE score of 19 points or higher, $\mathrm{n}=491$ ), the variable "depressive symptoms" was not included in the multivariate analysis.

The relative contribution of the different factors to the occurrence of lower respiratory tract infections was determined by population attributable risks (PAR), which combine the relative risk and prevalence of the different factors.

Data analyses were conducted using SPSS for Windows, V.12.0 (SPSS, Chicago, Illinois, USA).

\section{RESULTS}

\section{Study population}

Between September 1997 and September 1999, 705 participants were eligible for participation in the Leiden 85-plus Study. Ninety-two participants refused to participate and 14 participants died before enrolment, resulting in a study population of 599 participants (response rate of $87 \%$ ). ${ }^{25}$

For the present study, 12 participants for which valid clinical information about lower respiratory tract infections was missing were excluded. Therefore, our study population consisted of 587 persons. By the end of the follow-up period, there were still 273 participants aged 90 years (fig 1). During the 5 year follow-up period, 50 individuals refused further participation in the study and 264 individuals died. Primary causes of death were completed for 263 of the 264 participants. In total, $39.5 \%$ died as a result of cardiovascular causes (ICD-10 I00-99) and 60.5\% died from non-cardiovascular causes (all

Table 2 Incidences of lower respiratory tract infections in index and reference groups with the corresponding hazard ratios after univariate Cox regression analysis

\begin{tabular}{|c|c|c|c|c|}
\hline & $\mathbf{n}$ & $\begin{array}{l}\text { Incidence in index } \\
\text { group, per } 1000 \text { py } \\
(95 \% \mathrm{Cl})\end{array}$ & $\begin{array}{l}\text { Incidence in } \\
\text { reference group*, } \\
\text { per } 1000 \text { py } \\
(95 \% \mathrm{CI})\end{array}$ & HR $(95 \% \mathrm{CI})$ \\
\hline \multicolumn{5}{|l|}{ Sociodemographic factors } \\
\hline Male & 199 & $128.5(98.4$ to 158.6$)$ & $79.2(63.9$ to 94.5$)$ & 1.55 (1.14 to 2.10$)$ \\
\hline Institutionalised $\dagger$ & 104 & $153.0(105.0$ to 201.1$)$ & $84.3(70.0$ to 98.5$)$ & 1.73 (1.21 to 2.48$)$ \\
\hline Minimum income & 93 & $92.8(57.8$ to 127.8$)$ & $93.4(78.1$ to 108.7$)$ & $1.00(0.66$ to 1.50$)$ \\
\hline Only primary school & 380 & $101.6(83.3$ to 120.0$)$ & 79.6 (58.3 to 100.8$)$ & $1.27(0.92$ to 1.76$)$ \\
\hline Smoking, current or previous & 278 & $133.0(107.5$ to 158.6$)$ & 63.5 (48.2 to 78.9$)$ & $2.00(1.47$ to 2.73$)$ \\
\hline No yearly influenza vaccine $<85$ y & 133 & $79.3(52.6$ to 105.9$)$ & 97.1 (80.4 to 113.8$)$ & $0.82(0.56$ to 1.20$)$ \\
\hline \multicolumn{5}{|l|}{ Functioning } \\
\hline Severe cognitive impairment, MMSE $<19$ & 96 & $178.9(122.0$ to 235.7$)$ & $82.7(68.7$ to 96.6$)$ & 2.10 (1.46 to 3.02$)$ \\
\hline Depressive symptoms, GDS-15 >4 & 69 & $126.3(79.5$ to 173.1$)$ & $75.8(61.5$ to 90.2$)$ & $1.61(1.06$ to 2.44$)$ \\
\hline Disability in daily living & 201 & $147.0(113.8$ to 180.3$)$ & $73.9(59.3$ to 88.5$)$ & 1.94 (1.43 to 2.62$)$ \\
\hline \multicolumn{5}{|l|}{ Comorbidities and medication use } \\
\hline Diabetes mellitus & 85 & $95.6(56.5$ to 134.7$)$ & $93.3(78.4$ to 108.3$)$ & $1.00(0.65$ to 1.56$)$ \\
\hline Hypertension & 226 & $101.5(77.9$ to 125.1$)$ & 89.0 (71.8 to 106.3$)$ & $1.13(0.84$ to 1.53$)$ \\
\hline Myocardial infarct & 62 & $118.0(64.9$ to 171.1$)$ & $91.1(76.7$ to 105.6$)$ & 1.26 (0.78 to 2.03 ) \\
\hline Stroke & 59 & $206.2(129.9$ to 282.6$)$ & $85.2(71.3$ to 99.0$)$ & 2.26 (1.50 to 3.39$)$ \\
\hline Heart failure & 74 & $115.6(66.1$ to 165.0$)$ & $90.3(75.9$ to 104.8$)$ & $1.23(0.78$ to 1.94$)$ \\
\hline Chronic obstructive pulmonary disease & 69 & $270.6(184.5$ to 356.6$)$ & $79.2(65.8$ to 92.6$)$ & $3.07(2.13$ to 4.41$)$ \\
\hline Cancer & 104 & $110.6(71.7$ to 149.5$)$ & $91.0(76.1$ to 106.0$)$ & 0.86 (0.58 to 1.27$)$ \\
\hline Anaemia§ & 149 & $106.3(74.5$ to 138.0$)$ & $87.2(71.5$ to 102.9$)$ & 1.19 (0.84 to 1.69$)$ \\
\hline Oral glucocorticosteroid use & 11 & 266.3 (69.0 to 463.5$)$ & $91.5(77.6$ to 105.4$)$ & 2.77 (1.30 to 5.89$)$ \\
\hline Creatinine clearance $<30 \mathrm{ml} / \mathrm{min}$ & 41 & $121.6(52.8$ to 190.4$)$ & $89.5(75.0$ to 104.0$)$ & $1.35(0.75$ to 2.44$)$ \\
\hline Urea $>7.5 \mathrm{mmol} / \mathrm{l}$ & 233 & 105.3 (80.8 to 129.8 ) & 83.1 (66.0 to 100.2$)$ & $1.23(0.90$ to 1.68$)$ \\
\hline $\mathrm{CRP}>5 \mathrm{mg} / \mathrm{l}$ & 188 & 138.7 (105.7 to 171.7$)$ & 73.5 (58.7 to 88.4$)$ & 1.82 (1.33 to 2.49$)$ \\
\hline \multicolumn{2}{|c|}{ Overall incidence of lower respiratory tract infections } & 93.8 (79.8 to 107.7$)$ & & \\
\hline
\end{tabular}


Table 3 Factors associated with increased risk of developing lower respiratory tract infections after age 85 years, by multivariate Cox regression analysis

\begin{tabular}{ll}
\hline & HR (95\% Cl) \\
\hline Sociodemographic factors & \\
Male & $1.3(0.90$ to 1.9$)$ \\
Institutionalised & $1.1(0.72$ to 1.7$)$ \\
Smoking, current or previous & $1.9(1.3$ to 2.8$)$ \\
Functioning & \\
$\quad$ Severe cognitive impairment, MMSE $<19$ & $1.7(1.1$ to 2.8$)$ \\
Disability in daily living† & $1.6(1.1$ to 2.3$)$ \\
Comorbidities and medication use & \\
Chronic obstructive pulmonary disease & $3.4(2.3$ to 5.0$)$ \\
Stroke & $1.7(1.1$ to 2.7$)$ \\
Oral glucocorticosteroid use & $2.3(1.0$ to 5.0$)$ \\
CRP $>5$ mg/l & $1.4(1.0$ to 1.9$)$ \\
\hline
\end{tabular}

*Institutionalised refers to nursing or retirement home resident.

†Disability in daily living = unable to do any one of nine basic activities of daily living independently, according to the Groningen Activity Restriction Scale.

CRP, C reactive protein; HR, hazard ratio; MMSE, Mini-Mental State Examination.

other ICD-10 codes). Of those, 17 participants had infection as a primary cause of death, of whom 16 died from pneumonia (ICD-10 J17). Of the deceased participants, episodes of lower respiratory tract infections were registered until death occurred.

Table 1 shows the baseline characteristics of the study participants at the age of 85 years $(n=587)$. One-third of the population was male and $18 \%$ of participants were institutionalised. One-third of the population was restricted in their daily activities and over 15\% were severely cognitively impaired.

\section{Incidence of lower respiratory tract infections}

During 5 years of follow-up, 173 first episodes of lower respiratory tract infections were observed during 1845 person years at risk, resulting in an overall incidence of lower respiratory tract infections of 94 ( $95 \%$ confidence interval (CI) 80 to 108) per 1000 person years. During the follow-up period, 53 participants had recurrent lower respiratory tract infections ( $9 \%$ of total population, $31 \%$ of participants with lower respiratory tract infections).

Table 4 Population attributable risks (PAR) of the occurrence of lower respiratory tract infections after the age of 85 years, for factors that remained predictive after multivariate analysis

\begin{tabular}{ll}
\hline & PAR (\%) \\
\hline Sociodemographic factors & \\
Male $(\mathrm{n}=199)$ & 15 \\
Institutionalised $(\mathrm{n}=104)$ & 10 \\
Smoking, current or previous ( $\mathrm{n}=278)$ & 32 \\
Functioning & \\
$\quad$ Severe cognitive impairment, MMSE $<19(\mathrm{n}=96)$ & 12 \\
$\quad$ Disability in daily living† ( $=201)$ & 21 \\
Comorbidities and medication use & \\
Stroke $(\mathrm{n}=59)$ & 9 \\
Chronic obstructive pulmonary disease $(\mathrm{n}=69)$ & 2 \\
Oral glucocorticosteroid use $(\mathrm{n}=11)$ & 11 \\
CRP $>5 \mathrm{mg} / \mathrm{L}(\mathrm{n}=188)$ &
\end{tabular}

* Institutionalised refers to nursing or retirement home resident.

$\dagger$ Disability in daily living = unable to do any one of nine basic activities of daily living independently, according to the Groningen Activity Restriction Scale.

CRP, C reactive protein; MMSE, Mini-Mental State Examination.

\section{Predictive factors of lower respiratory tract infections}

Table 2 shows the incidences of lower respiratory tract infections in various groups for all selected potentially predictive factors with concomitant univariate hazard ratios (HRs). The occurrence of lower respiratory tract infections was associated with (listed from highest to lowest HR) COPD, oral glucocorticosteroid use, medical history of stroke, severe cognitive impairment, smoking, disability in daily living, CRP $>5 \mathrm{mg} / \mathrm{l}$, institutionalisation, depressive symptoms and male gender (table 2). Additional analyses showed that previous smokers $(\mathrm{n}=186)$ had a $1.92(95 \%$ CI 1.37 to 2.68$)$ increased risk of developing a lower respiratory tract infection compared with participants who had never smoked; for current smokers $(\mathrm{n}=92)$ this risk was 2.17 (95\% CI 1.42 to 3.29$)$. The occurrence of lower respiratory tract infections was not associated with socioeconomic status (measured by income and educational level), renal function (measured by creatinine clearance and plasma level of urea) or diabetes mellitus (table 2). Participants who had no influenza vaccination in the year before age 85 years had similar risks of developing lower respiratory tract infections as those who had an influenza vaccination (HR 0.82, 95\% CI 0.56 to 1.20 ).

After multivariate analysis, the following predictive factors remained significantly associated with the occurrence of lower respiratory tract infections (table 3): COPD, oral glucocorticosteroid use, current and previous smoking, severe cognitive impairment, medical history of stroke and disability in daily living.

\section{Population attributable risk}

To further assess the relative contribution of the various factors to the occurrence of lower respiratory tract infections in the general population from age 85 years onwards, the PAR of each variable that remained predictive of lower respiratory tract infections in the multivariate analysis was determined (table 4). The highest PARs were revealed for current and previous smoking (32\%), disability (21\%), male gender (15\%) and COPD $(15 \%)$.

\section{DISCUSSION}

In this population based prospective follow-up study of the very elderly, the incidence of lower respiratory tract infections was 94 per 1000 person years at risk. COPD, oral glucocorticosteroid use, smoking, severe cognitive impairment, medical history of stroke and disability in daily living were among the strongest predictive factors for developing lower respiratory tract infections between the ages of 85 and 90 years. The population attributable risk was highest for smoking (32\%).

In previous studies, the incidence of lower respiratory tract infections (defined as pneumonia and acute bronchitis) in persons aged 65-74 years was 42 per 1000 person years, ${ }^{2}$ and increased to 70 per 1000 person years among persons aged 75 years and over. ${ }^{6}$ In the present study, in persons from age 85 years onwards, the incidence of lower respiratory tract infections was 94 per 1000 person years. Thus the outcome of our study corroborates these earlier findings and supports the hypothesis that the incidence of lower respiratory tract infections increases with age, even in the higher age ranges. ${ }^{3-5}$

It is well known that patients with COPD have a higher rate of lower respiratory tract infections than those without COPD. ${ }^{3-5}{ }^{11} 26-28$ Indeed, in our study population, the presence of COPD was a predictor of lower respiratory tract infections. Interestingly, however, smoking carries an equal risk and 
contributed even stronger to lower respiratory tract infections in our study. It is possible that clinically undiagnosed COPD in elderly current and previous smokers underlies part of these associations. An additional likely explanation is that smoking impairs immunological processes in the lung and directly causes an increased risk of respiratory tract infections in smokers. ${ }^{29-31}$

In our study, factors reflecting declined functional status (severe cognitive impairment and disability in daily living) were also found to be predictors of lower respiratory tract infections from the age of 85 years onwards. Our results confirm the findings of the study by Lipsky et al who found a relation between both dementia and stroke and pneumococcal pneumonia in patients aged $50-80$ years. ${ }^{5}$ Since more than one-third of our population based sample was restricted in their functioning, declined functional status importantly contributes to the occurrence of lower respiratory tract infections in the general population, reflected by a total PAR of $33 \%$.

Lower respiratory tract infection is a known complication of clinical influenza infection, especially in the elderly. With influenza vaccination, the incidence of both influenza and its complications is lowered. Therefore, influenza vaccination will be offered particularly to the elderly at risk for lower respiratory tract infections. Interestingly, in this study, we found that participants who had no influenza vaccination in the year before age 85 years had similar risks of developing lower respiratory tract infections as those who had received a vaccination (HR $0.82,95 \%$ CI 0.56 to 1.20 ). This may be a positive vaccination effect as those elderly patients who received the influenza vaccination were expected to have a higher risk of lower respiratory tract infections.

Although diabetes mellitus is reportedly associated with a greater predisposition to infections, ${ }^{32-35}$ we did not find any association between diabetes mellitus and lower respiratory tract infections. This finding is in line with the findings of three other investigations on this topic. ${ }^{3511}$ Recently, a Dutch study showed that patients with type 2 diabetes mellitus had a higher risk of lower respiratory tract infections compared with patients with hypertension (odds ratio 1.3 (95\% CI $1.1-1.5)$ ). ${ }^{35}$ Differences in age, type of diabetes and definition of lower respiratory tract infections possibly underlie the inconsistency in our data and might also explain the inconsistent findings.

The present study is a unique population based sample of participants aged 85 years and over, with extensive baseline measurement and almost complete follow-up for morbidity and mortality. To our knowledge, we are the first to examine the incidence and predictive factors of lower respiratory tract infections in a large group of unselected 85-year-olds in a population based setting.

It may be considered as a limitation of our study that lower respiratory tract infections were not diagnosed by standardised diagnostic procedures, including chest radiography, sputum and blood cultures. In our study, however, all lower respiratory tract infections were clinically diagnosed by general practitioners and nursing home physicians based on medical history taking, physical examination and clinical judgment during a consultation with the patient. In the preparation phase of the Leiden 85plus Study, we decided to measure lower respiratory tract infections instead of pneumonia because this latter diagnosis is generally known to be inaccurate in elderly patients in primary care, as diagnostic procedures such as chest radiography, sputum and blood cultures are not usually carried out. ${ }^{3}$ Because of this design, we do not have any information about the incidence of the subdiagnosis viral pneumonia, bacterial pneumonia or acute bronchitis. This procedure, however, reflects usual primary care and enables generalisation of our results to daily care.

What is the clinical importance of our findings? As most developed countries face population aging and the incidence of lower respiratory tract infections increases with age, it is important to consider preventive and early clinical management strategies for lower respiratory tract infections in the very elderly. For the very elderly at high risk of lower respiratory tract infections, two strategies of prevention are possible. Firstly, elderly with changeable risk factors could be advised to reduce exposure to these factors; very elderly who currently smoke could be advised to stop or reduce smoking, and unnecessary use of glucocorticoids could be reduced. Secondly, those very elderly at risk could be firmly advised to apply for influenza and pneumococcal vaccination. Before giving this advice and changing current guidelines, however, the effects of both strategies on reducing lower respiratory tract infections in very elderly people have to be studied in randomised intervention studies.

\section{Competing interests: None}

Ethics approval: The Medical Ethics Committee of Leiden University Medical Centre approved the study.

\section{REFERENCES}

1. Seppä Y, Bloigu A, Honkanen PO, et al. Severity assessment of lower respiratory tract infection in elderly patients in primary care. Arch Intern Med 2001;161:2709-13.

2. Bont $\mathbf{J}$, Hak E, Hoes AW, et al. A prediction rule for elder primary care patients with lower respiratory tract infections. Eur Respir J 2007;29:969-75.

3. Farr BM, Woodhead MA, Macfarlane JT, et al. Risk factors for community-acquired pneumonia diagnosed by general practitioners in the community. Respir Med 2000;94:422-7.

4. Koivula I, Sten M, Mäkelä PH. Risk factors for pneumonia in the elderly. Am J Med 1994;96:313-20.

5. Lipsky BA, Boyko EJ, Inui TS, et al. Risk factors for acquiring Pneumococcal infections. Arch Intern Med 1986;146:2179-85.

6. Hak E, Rovers MM, Kuyvenhoven MM, et al. Incidence of GP-diagnosed respiratory tract infections according to age, gender and high risk co-morbidity: the Second Dutch National Survey of General Practice. Fam Pract 2006;23:291-4.

7. Posfay-Barbe KM, Wald ER. Pneumococcal vaccines: do they prevent infection and how? Curr Opin Infect Dis 2004;17:177-84.

8. Bogaert D, Hermans PWM, Adrian PV, et al. Pneumococcal vaccines: an update on current strategies. Vaccine 2004;22:2209-20.

9. Vila-Córcoles A, Ochoa-Gondar 0, Hospital I, et al. Protective effects of the 23valent pneumococcal polysaccharide vaccine in the elderly population: the EVAN-65 study. Clin Infect Dis 2006;43:860-8.

10. Christenson B, Hedlund J, Lundbergh P, et al. Additive preventive effect of influenza and pneumococcal vaccines in elderly persons. Eur Respir $\mathrm{J}$ 2004;23:363-8.

11. Almirall J, Bolíbar I, Balanzó X, et al. Risk factors for community-acquired pneumonia in adults: a population-based case-control study. Eur Respir $J$ 1999;13:349-55.

12. Bauer TT, Ewig S, Marre R, et al. CRB-65 predicts death from community-acquired pneumonia. J Intern Med 2006:260:93-101.

13. Riquelme R. Torres A, El-Ebiary $M$, et al. Community-acquired pneumonia in the elderly: a multivariate analysis of risk and prognostic factors. Am J Respir Crit Care Med 1996;154:1450-5.

14. Fernandez-Sabe N, Carratala J, Roson B, et al. Community-acquired pneumonia in very elderly patients: causative organisms, clinical characteristics, and outcomes. Medicine 2003;82:159-69.

15. Lim WS, Macfarlane JT. Defining prognostic factors in the elderly with CAP: a case controlled study of patients aged $\geqslant 75$ yrs. Eur Respir J 2001;17:200-5.

16. Naito T, Suda T, Yasuda K, et al. A validation and potential modification of the pneumonia severity index in elderly patients with community-acquired pneumonia. J Am Geriatr Soc 2006;54:1212-19.

17. Brancati FL, Chow JW, Wagener MM, et al. Is pneumonia really the old man's friend? Two-year prognosis after CAP. Lancet 1993:342:30-3.

18. Conte HA, Chen YT, Mehal W, et al. A prognostic rule for elderly patients admitted with community-acquired pneumonia. Am J Med 1999;106:20-8.

19. Fine MJ, Smith MA, Carson CA, et al. Prognosis and outcomes of patients with community-acquired pneumonia. A meta-analysis. JAMA 1996;275:134-41.

20. Lim WS, Lewis S, Macfarlane JT. Severity prediction rules in CAP: a validation study Thorax 2000;55:219-23.

21. Bootsma-van der Wiel A, Gussekloo J, de Craen AJM, et al. Disability in the oldest old: "can do" or "do do"? J Am Geriatr Soc 2001;49:909-14. 
22. Guidelines for ATC Classification and DDD Assignment. Oslo, Norway: World Health Organization Collaborating Centre for Drug Statistics Methodology, 1996.

23. Nutritional anaemias. Report of a WHO scientific group. World Health Organ Tech Rep Ser 1968:405:5-37.

24. Cockcroft DW, Gault MH: Prediction of creatinine clearance from serum creatinine. Nephron 1976;16:31-41.

25. der Wiel $\mathbf{A B}$, van Exel $\mathrm{E}$, de Craen AJ, et al. A high response is not essential to prevent selection bias: results from the Leiden 85-plus study. J Clin Epidemiol 2002; 55:1119-25.

26. Monto AS, Millicent WH, Ross HW. The Tecumseh study of respiratory illness. VIII. Acute infection in chronic respiratory disease and comparison groups. Am Rev Respir Dis 1975;111:27-36

27. McNamara MJ, Phillips IA, Williams OB. Viral and Mycoplasma pneumoniae infections in exacerbations of chronic lung disease. Am Rev Respir Dis 1969:100:19-24.

28. Westerberg SC, Smith CB, Renzetti AD. Mycoplasma infections in patients with chronic obstructive pulmonary disease. J Infect Dis 1973;127:491-7.
29. Stringer KA, Tobias M, O'Neill HC, et al. Cigarette smoke extract-induced suppression of caspase-3-like activity impairs human neutrophil phagocytosis. Am J Physiol Lung Cell Mol Physiol 2007;292:L1572-9.

30. Baik I, Curhan GC, Rimm EB, et al. A prospective study of age and lifestyle factors in relation to community-acquired pneumonia in US men and women. Arch Intern Med 2000;160:3082-8.

31. Klement E, Talkinton DF, Wasserzug 0 , et al. Identification of risk factors for infection in an outbreak of Mycoplasma pneumoniae respiratory tract disease. Clin Infect Dis 2006:43:1239-45.

32. Shah BR, Hux JE. Quantifying the risk of infectious disease for people with diabetes. Diabetes Care 2003;26:510-13.

33. Calvet HM, Yoshikawa TT. Infections in diabetes. Infect Dis Clin North Am 2001;15:407-21.

34. Pozzilli P, Leslie RDG. Infections and diabetes: mechanisms and prospects for prevention. Diabet Med 1994;11:935-41.

35. Muller LM, Gorter KJ, Hak E, et al. Increased risk of infection in patients with diabetes mellitus type 1 or 2. Ned Tijdschr Geneeskd 2006;150:549-553.

\section{Lung alert}

\section{Lung transplantation in patients with COPD}

Both unilateral and bilateral lung transplantation in chronic obstructive pulmonary disease (COPD) can improve pulmonary function, exercise capacity and quality of life; however, whether lung transplantation actually improves survival is debated.

In this study, survival after transplantation of one or two lungs in end-stage COPD in nearly 10000 patients in the International Society for Heart and Lung Transplantation (ISHLT) registry was compared. This observational study included all reported lung transplantations worldwide from inception of the registry in 1987 until 2006. Median survival time after bilateral lung transplantation (6.41 years) was better than after unilateral procedures (4.59 years). However, bilateral lung transplantation had little benefit compared with unilateral transplantation for patients aged $\geqslant 60$ years.

Lung transplants are very limited in supply and therefore more concentration on preventing the disease through smoking prevention programmes and other conventional treatments beside lung volume reduction, either surgically or with bronchoscopic placement of one-way endobronchial valves, may still be a better option. Although rigorous application of sophisticated statistical methods was applied to adjust for baseline differences, unmeasured differences might exist between recipients of unilateral and bilateral transplants and only a randomised controlled trial will remove all possible confounders.

- Thabut G, Christie JD, Ravaud P, et al. Survival after bilateral versus single lung transplantation for patients with chronic obstructive pulmonary disease: a retrospective analysis of registry data. Lancet 2008;371:744-51.

\section{A Benrajab}

Correspondence to: Dr A Benjarab, Specialist Registrar, Royal Albert Edward Infirmary, Wigan, Lancashire, UK; abenrajab@ gmail.com 